Sustainable and Reusable Gelatin-based Hydrogel "Jelly Ice Cubes" as

Food Coolant. I: Feasibilities and Challenges

\author{
Jiahan Zou ${ }^{\dagger}$, Luxin Wang $\ddagger$, Gang Sun*, \\ † Department of Biological and Agricultural Engineering, ‡ Department of Food Science and Technology, \\ University of California, One Shields Avenue, Davis, California, 95616, United States
}

\title{
AUTHOR INFORMATION
}

\section{Corresponding Author}

Gang Sun* E-mail: gysun@ucdavis.edu. Tel: (530) 752-0840

\section{Supporting Information}

\section{Page S1-S2}

Figure S1. (a) The normal pressure produced by the food load on top of JICs. (b) The representative compressive curve of $10 \%$ gelatin hydrogel (JIC-FTC0).

Figure S2. The schematic drawings of the freeze-thaw treatment JICs.

Figure S3. Photos of JICs after five times of 50ppm bleach wash. 
a.

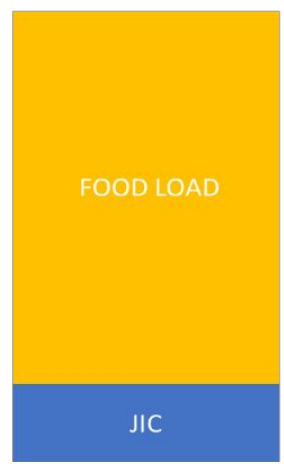

b.

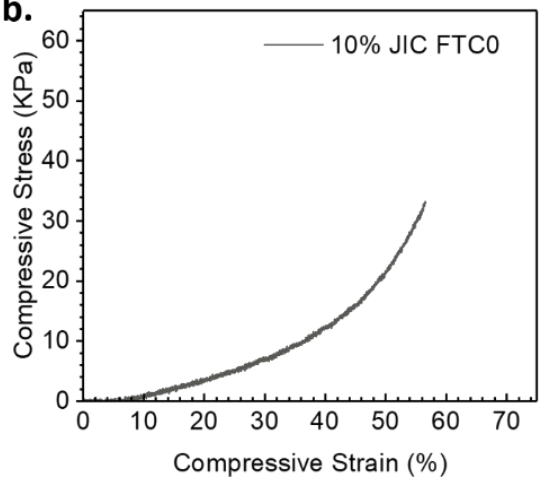

Figure S1. (a) The normal pressure produced by the food load on top of JICs. (b) The representative compressive curve of $10 \%$ gelatin hydrogel (JIC-FTC0).

Assume the food load has a uniform density $(\rho)$ with cuboid shape. $h(\mathrm{~m})$ represents the height of the food load

$$
\begin{gathered}
\rho=1000\left(\frac{\mathrm{kg}}{\mathrm{m}^{3}}\right) \\
g=9.8\left(\frac{\mathrm{N}}{\mathrm{kg}}\right) \\
P=\frac{F}{A}=\frac{m g}{A}=\frac{\rho V g}{A}=\frac{\rho A h g}{A}=\rho g h=9800 h\left(\frac{\mathrm{N}}{\mathrm{m}^{3}}\right) \approx 10 h\left(\frac{\mathrm{kPa}}{\mathrm{m}}\right)
\end{gathered}
$$

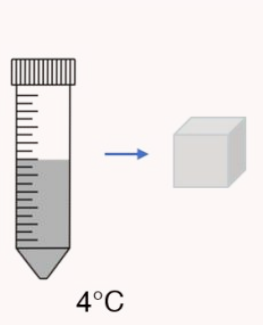

Sol-gel transformation

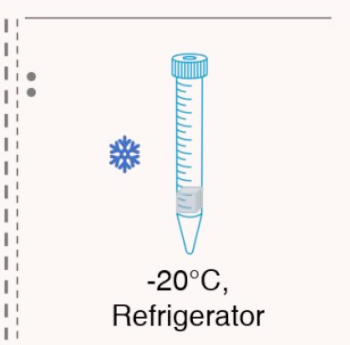

Freeze $(F)$

Freeze-Thaw Cycles $(\mathrm{FTC}=0,1, \ldots, 5)$

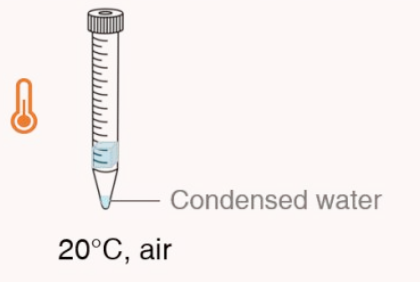

Thaw $(\mathrm{T})$

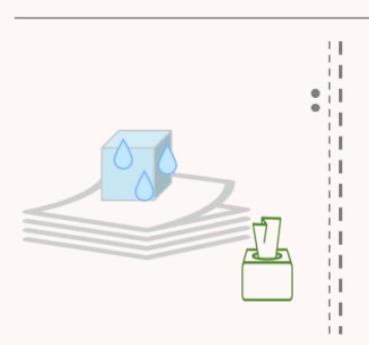

Dry surface condensed water

Figure S2. The schematic drawings of the freeze-thaw treatment JICs.
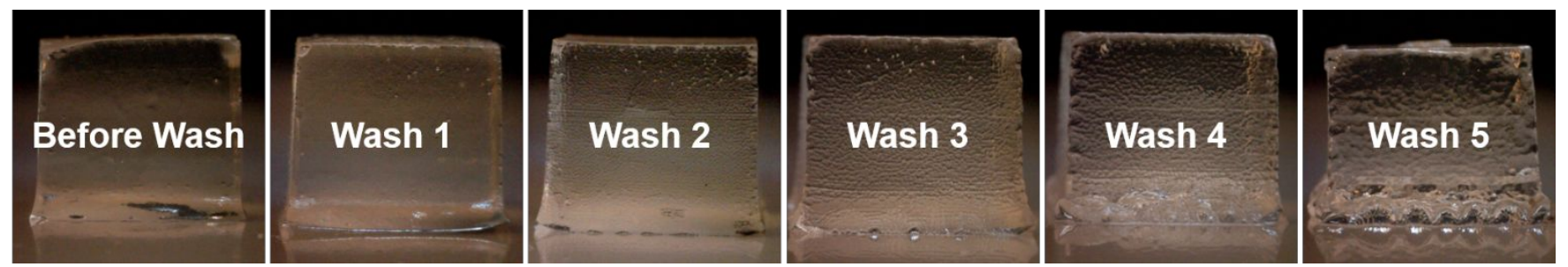
Figure S3. Photos of JICs after five times of 50ppm bleach wash. 